\title{
Quadris espásticos da paralisia cerebral - Estudo retrospectivo do salvamento com a cirurgia de McHale*
}

\section{Spastic Hips in Cerebral Palsy - Retrospective Study of Salvage with the McHale Procedure}

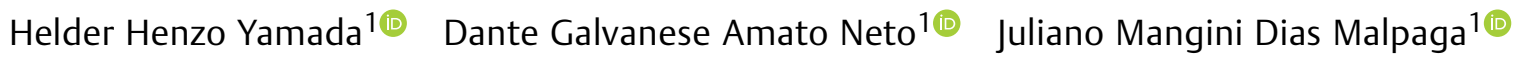 \\ Patricia Maria de Moraes Barros Fucs ${ }^{10}$ \\ ${ }^{1}$ Departamento de Ortopedia e Traumatologia, Faculdade de Ciências \\ Médicas, Santa Casa de Misericórdia de São Paulo, São Paulo, SP, Brasil \\ Endereço para correspondência Helder H. Yamada, Mestre, Rua \\ Dr. Cesário Motta Jr., 112, São Paulo, SP, Brasil \\ (e-mail: helderyamada@gmail.com; helder.henzo@gmail.com).
}

Rev Bras Ortop 2021;56(2):244-250.

\section{Resumo \\ Objetivo Fazer uma avaliação retrospectiva e transversal quanto à melhora da dor $\mathrm{e}$ do posicionamento de todos os pacientes portadores de paralisia cerebral (PC) espástica com deformidade grave no quadril submetidos ao procedimento de McHale em nosso centro. Secundariamente, objetivou-se analisar as possíveis complicações do procedimento. \\ Métodos Foram analisados todos os pacientes consecutivos tratados no período entre 1995 e 2017. Clinicamente, os pacientes deveriam apresentar dor à mobilização do quadril, dificuldade de posicionamento para se sentar e para os cuidados de higiene, e prontuário médico com dados completos; quanto ao grau de função motora, utilizou- se o Sistema de Classificação da Função Motora Grossa (Gross Motor Function Classification System, GMFCS, em inglês). A avaliação radiográfica no período pré- operatório analisou a porcentagem de migração (PM), o tipo de deformidade de acordo com a Escala de Classificação de Quadril na Paralisia Cerebral de Melbourne (Melbourne Cerebral Palsy Hip Classification Scale, MCPHS), e a deformidade da cabeça femoral. No período pós-operatório, analisaram-se a presença de migração proximal do fragmento \\ Palavras-chave \\ - paralisia cerebral \\ - quadril/deformidades \\ - quadril/cirurgia do fêmur proximal, as alterações e/ou a falha do implante utilizado, e a possível ossificação heterotópica. Consideraram-se como desfechos: D1- satisfatório: remissão da dor, mobilidade indolor, melhora do posicionamento; e D2- insatisfatório: falha no procedimento, que necessitou de reabordagem cirúrgica.}

\footnotetext{
Estudo realizado no Grupo das Doenças Neuromusculares, Departamento de Ortopedia e Traumatologia, Faculdade de Ciências Médicas da Santa Casa de São Paulo, Santa Casa de Misericórdia de São Paulo, São Paulo, Brasil.
}

recebido

17 de Setembro de 2019

aceito

15 de Abril de 2020

Publicado online

Setembro 22, 2020
DOI https://doi.org/ $10.1055 / \mathrm{s}-0040-1713391$ ISSN 0102-3616.

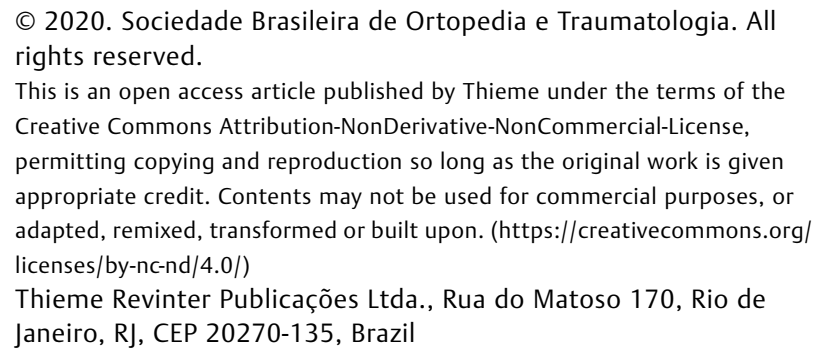

(c) 2020. Sociedade Brasileira de Ortopedia e Traumatologia. All rights reserved.

This is an open access article published by Thieme under the terms of the Creative Commons Attribution-NonDerivative-NonCommercial-License, permitting copying and reproduction so long as the original work is given appropriate credit. Contents may not be used for commercial purposes, or adapted, remixed, transformed or built upon. (https://creativecommons.org/ licenses/by-nc-nd/4.0/) Thieme Revinter Publicações Ltda., Rua do Matoso 170, Rio de Janeiro, RJ, CEP 20270-135, Brazil 


\section{Abstract}

Keywords

- cerebral palsy

- hip/deformities

- hip/surgery
Resultados No total, 47 pacientes (53 quadris) foram tratados. Funcionalmente, quanto à classificação no GMFCS, 43 pacientes eram GMFCS V (91\%), 3 pacientes eram GMFCS IV (6\%), e 1 paciente era GMFCS III (2\%). A média da idade foi de 13 anos e 2 meses. $O$ tempo de seguimento variou de 1 ano a 15 anos e 4 meses, com média de 4 anos e 8 meses. Quanto ao desfecho da cirurgia de McHale, ele foi satifatório (D1) em 36 pacientes (41 quadris), perfazendo 77\% dos nossos casos, e insatisfatório (D2) em 11 (23\%) casos.

Conclusão A cirurgia de McHale é uma opção no tratamento para os níveis IV e V, mas devemos estar alertas para as possíveis complicações.

Objective To perform a retrospective and cross-sectional assessment to determine the pain and positional improvement of all patients with spastic cerebral palsy (CP) and severe hip deformity who underwent a McHale procedure in our center. A second objective was to analyze the potential complications from the procedure.

Methods All consecutive patients treated between 1995 and 2017 were analyzed. Clinically, the patients should present pain on hip mobilization, difficulty in positioning for sitting and hygiene care, and medical records with complete data; functionally was assessed through the Gross Motor Function Classification System (GMFCS). In the preoperative radiographs, we analyzed the migration percentage (MP), the type of deformity according to the Melbourne Cerebral Palsy Hip Classification Scale (MCPHCS), and the type of deformity of the femoral head. After the surgery, we assessed the proximal migration of the proximal femoral fragment, implant changes and/or failure, and potential heterotopic ossification. The outcomes were reported as successful (D1) in patients presenting remission of pain, painless mobility, and improved positioning, or unsuccessful (D2) in those presenting procedural failure that required a new surgery.

Results In total, 47 patients (53 hips) were treated. Functionally, 43 patients were classified as GMFCS V (91\%), 3 as GMFCS IV patients (6\%), and 1 as GMFCS III (2\%). The mean age was 13 years and 2 months. The follow-up ranged from 1 year to 15 years and 4 months, with an average of 4 years and 8 months. A total of 36 patients ( 41 hips) presented successful (D1) outcomes after the McHale surgery, corresponding to $77 \%$ of our cases, whereas 11 (23\%) cases had unsuccessful (D2) outcomes.

Conclusion The McHale surgery is a treatment option for GMFCS IV and V, but we must be aware of the potential complications.

\section{Introdução}

As deformidades no quadril ocorrem em mais de um terço das crianças com Paralisia Cerebral (PC), e são a segunda deformidade mais comum após o pé em equino. ${ }^{1-5}$

A "doença do quadril espástico" é secundária ao desequilíbrio muscular, atuando em um esqueleto em crescimento. Atitudes em flexão e adução vão progressivamente se tornando contraturas até que a deformidade fica estabelecida. A deformidade em flexão e adução e a limitação da mobilidade articular impedem ou dificultam os cuidados gerais de higiene e de posicionamento sentado, o que torna a qualidade de vida muito comprometida nestes pacientes. Secundariamente na articulação, as alterações degenerativas podem se tornar dolorosas, o que agrava o quadro destes pacientes. ${ }^{1,2}$
O objetivo do tratamento nas deformidades do quadril espástico é mantê-lo móvel, bem locado, sem dor, e com arco de movimento simétrico em relação ao contralateral. ${ }^{1,2,5}$

Quando estabelecidas a subluxação ou a luxação, há indicação cirúrgica de reconstrução desses quadris. ${ }^{2,4,6-13}$ Porém, nos casos em que já existe deformidade da cabeça femoral e incongruência articular, a oportunidade da reconstrução foi perdida. Nestes casos, há a indicação dos procedimentos chamados de "salvamento". Estes podem ser: artroplastia de ressecção do fêmur proximal, ${ }^{14}$ osteotomia em valgo do fêmur proximal com ou sem ressecção da cabeça e do colo do fêmur (cirurgia de McHale), ${ }^{15,16}$ artrodese do quadrill, ${ }^{17}$ artroplastia total do quadril, ${ }^{18}$ e artroplastia de interposição de prótese de fêmur proximal. ${ }^{1,13}$

Ainda hoje, após várias revisões da literatura sobre o assunto, ${ }^{19-21}$ não há um consenso sobre o melhor tratamento nesses casos, especialmente porque as publicações 
são geralmente de séries de casos cuja falta de uniformidade torna a comparação difícil, levando-se em conta as condições do paciente e suas comorbidades e o meio onde o paciente é tratado.

Sabe-se que a presença de dor associada a deformidade do quadril nos pacientes espásticos tem relação direta com a sua qualidade de vida. ${ }^{1,2,12}$ O principal motivo do tratamento nos pacientes portadores de PC espástica com deformidades nos quadris sintomáticas e graves, não mais passíveis de cirurgia reconstrutora, é melhorar os sintomas dolorosos e facilitar o posicionamento.

O objetivo deste estudo foi fazer a avaliação retrospectiva e transversal quanto à melhora da dor e do posicionamento de todos os pacientes portadores de PC espástica com deformidade grave no quadril submetidos ao procedimento de McHale ${ }^{15}$ em nosso centro. Secundariamente, objetivouse analisar as possíveis complicações do procedimento.

\section{Casuística e Métodos}

O estudo foi aprovado pela Comissão de Ética do hospital (CAAE 94352318.9.0000.5479), e os responsáveis legais pelos pacientes assinaram os termos de consentimento livre e esclarecido para a inclusão dos dados clínicos neste estudo.

Foram analisados todos os pacientes consecutivos submetidos à cirurgia de McHale pelo Grupo de Doenças Neuromusculares da nossa instituição entre 1995 e 2017. Os critérios de inclusão foram: pacientes portadores de PC espástica com quadril subluxado ou luxado, e com deformidade da cabeça femoral que inviabilizou a reconstrução articular. Clinicamente, os pacientes deveriam apresentar dor à mobilização do quadril, dificuldade de posicionamento para se sentar e para os cuidados de higiene, e prontuário médico com dados completos. Foram excluídos os pacientes com dados incompletos e os que não retornaram nas avaliações ambulatoriais.

Para a avaliação do nível da dor dos pacientes no período pré-operatório, não foi utilizada escala objetiva: dados dos prontuários informaram que todos os pacientes apresentavam dor à mobilização do quadril acometido e/ou dificuldades ou intolerância no posicionamento sentado no período pré-operatório. Uma vez que se trata de avaliação retrospectiva, considerou-se a melhora relatada pelo paciente e/ou cuidador no último retorno ambulatorial, sendo que os pacientes seguem em acompanhamento no nosso serviço.

A avaliação radiográfica foi realizada nos períodos préoperatório e na última avaliação ambulatorial, constando de radiografia em incidência anteroposterior da bacia, panorâmica da coluna vertebral, e nas incidências posteroanterior e lateral. No período pré-operatório, a análise radiográfica da bacia foi realizada utilizando-se como parâmetros a porcentagem de migração (PM), ${ }^{22}$ o tipo de deformidade de acordo com a Escala de Classificação de Quadril na Paralisia Cerebral de Melbourne (Melbourne Cerebral Palsy Hip Classification Scale, MCPHPS), ${ }^{5}$ e o tipo da deformidade da cabeça femoral. ${ }^{1}$ No período pós-operatório, também foi verificada a presença de migração proximal do fragmento do fêmur proximal, as alterações e/ou a falha do implante utilizado, e a possível ossificação heterotópica. Na radiografia da coluna vertebral, na incidência posteroanterior, em decúbito dorsal, foi medido o ângulo de Cobb apenas para o diagnóstico de escoliose, sendo considerada a curva com gravidade moderada nas angulações $\geq 40^{\circ} .{ }^{23}$ Para a mensuração dos ângulos e distâncias, foi utilizado o programa MB-Ruler, versão 5.3 para Windows (MB-Softwaresolutions, Iffezheim, Alemanha), com até 2 casas decimais. As medidas radiográficas foram feitas por dois avaliadores experientes e independentes, e foi calculado o coeficiente de correlação intraclasse (CCI) entre observadores.

Procedimentos cirúrgicos haviam sido realizados previamente em 8 pacientes ( 15 quadris), sendo em 7 , tenotomia dos adutores bilateral, e, em 1, redução cruenta do quadril associada a osteotomia varizante e de rotação externa do fêmur proximal, e a osteotomia pélvica de Dega, unilateral, realizada em outro serviço.

A técnica cirúrgica, de acordo com a descrição original (McHale et al. ${ }^{15}$ ), constitui os seguintes tempos: com o paciente em decúbito dorsal horizontal, inicialmente é realizada a tenotomia dos adutores pela via deles; o quadril é exposto por meio da via anterolateral (Watson-Jones). Com a cabeça femoral exposta, uma osteotomia é feita na base do colo femoral usando uma serra de nitrogênio. A cabeça femoral é removida, enquanto o ligamento redondo é preservado dentro do acetábulo. Uma cunha óssea de base lateral é removida do fêmur proximal no nível do trocânter menor para promover abdução em torno de $45^{\circ}$. A fixação da osteotomia se dá mediante o uso de placa reta de compressão dinâmica (PDC) ou placa de compressão de bloqueio (PCB) (Synthes, Solothurn, Suíça), moldada para a abdução do fragmento distal em média de $45^{\circ}$. O pequeno trocanter é deslocado para o acetábulo, e o ligamento redondo é então suturado no tendão do músculo psoas. Procede-se à capsulorrafia, sendo que a parte inferior da cápsula em geral não pode ser fechada. A sutura é por planos, e não é utilizada imobilização pós-operatória. ${ }^{15}$

No período pós-operatório, as complicações foram divididas em menores e maiores, sendo as menores definidas como: persistência da dor relacionada ao implante com ou sem sua exposição; fraturas do membro inferior operado; e a presença de ossificação heterotópica. As maiores complicações consideradas neste estudo foram: necessidade de internação; e submissão a outro procedimento cirúrgico.

A avaliação final considerou dois desfechos: o primeiro, satisfatório (D1), nos pacientes que permaneceram bem (com menos dor e sentados) após o procedimento, e os pacientes que apresentaram complicações consideradas menores; o segundo, insatisfatório (D2), para pacientes que apresentaram complicações maiores e que posteriormente foram submetidos a novo procedimento cirúrgico, no caso, a cirurgia de Castle. ${ }^{14}$

\section{Resultados}

No período do estudo, foram tratados 57 pacientes com 65 quadris operados. Foram excluídos 10 pacientes (12 quadris) que não retornaram para reavaliação, restando 47 pacientes (53 quadris), que representaram o grupo em estudo. 
Tabela 1 Dados demográficos dos pacientes

\begin{tabular}{|c|c|}
\hline $\begin{array}{l}\text { Número de pacientes } \\
\text { (número de quadris) }\end{array}$ & $47(53)$ \\
\hline $\begin{array}{l}\text { Idade } \\
\text { (anos e meses) }\end{array}$ & $\begin{array}{l}\text { média: } 13+2(163.28) \\
\text { mínima }: 5+4(64) \\
\text { máxima : } 35+10(430)\end{array}$ \\
\hline Gênero & $\begin{array}{l}\text { masculino: } 19 \\
\text { feminino: } 28\end{array}$ \\
\hline GMFCS & $\begin{array}{l}\text { III }-1 \\
\text { IV }-3 \\
V-43\end{array}$ \\
\hline Cirugias prévias & $\begin{array}{l}8 \text { (15 quadris) } \\
7 \text { - tenotomia dos } \\
\text { adutores bilateral } \\
1 \text { - redução cruenta do } \\
\text { quadril + osteotomia } \\
\text { femoral e pélvica }\end{array}$ \\
\hline Presença de escoliose & $35(75 \%)$ casos \\
\hline $\begin{array}{l}\text { Tempo de seguimento } \\
\text { anos }+ \text { meses (meses) }\end{array}$ & $\begin{array}{l}\text { mínimo : } 1+0(12) \\
\text { máximo : } 15+4(184) \\
\text { média : } 4+8(56) \\
\text { mediana : } 3+10(46)\end{array}$ \\
\hline
\end{tabular}

Abreviatura: GMFCS, Gross Motor Function Classification System.

Tabela 2 Resultados radiográficos

\begin{tabular}{|l|l|l|}
\hline & & Porcentagem \\
\hline Porcentagem & $33 \%-89 \%$ - 6 casos & $11 \%$ \\
de migração & $>90 \%-47$ casos & $89 \%$ \\
\hline Tipo de deformidade & TIPO 1 - 10 & $19 \%$ \\
da cabeça femoral & TIPO 2 - 22 & $41 \%$ \\
& TIPO 3 - 21 & $40 \%$ \\
\hline Grau no MCPHCS & Grau 5-6 casos & $11 \%$ \\
& Grau 6-47 casos & $89 \%$ \\
\hline
\end{tabular}

Abreviatura: MCPHCS, Melbourne Cerebral Palsy Hip Classification Scale.

A distribuição do sexos foi de 19 pacientes do sexo masculino e 28 do feminino. Quanto à lateralidade, 15 pacientes tinham o quadril acometido do lado direito, 26, do esquerdo, e 6 casos eram bilaterais. De acordo com o Sistema de Classificação da Função Motora Grossa (Gross
Motor Function Classification System, GMFCS), ${ }^{24}$ foram 43 pacientes nível $\mathrm{V}(91 \%), 3$ pacientes IV (6\%), e um paciente III (2\%). A média da idade dos pacientes na ocasião da cirurgia foi de 13 anos e 2 meses (mediana de 12 anos e 8 meses), variando entre 5 anos e 4 meses e 35 anos e 10 meses de idade ( - Tabela $\mathbf{1}$ ).

O tempo de internação dos pacientes apresentou média de cinco dias, e o tempo de consolidação da osteotomia variou de seis a oito semanas.

O tempo de seguimento variou de 1 ano (12 meses) a 15 anos e 4 meses (184 meses), com média de 4 anos e 8 meses (56 meses) e mediana de 3 anos e 10 meses (46 meses).

Achados radiográficos: o CCI calculado para a avaliação radiográfica foi maior do que 0,80 , considerado excelente; portanto, somente a média aritmética e a mediana final foram utilizadas. A PM inicial variou de $60 \%$ a $100 \%$, com média de $96,75 \%$ e mediana de $100 \%$, sendo 6 quadris entre $33 \%$ e $89 \%$, e 47 quadris acima dos $90 \%$. O MCPHCS foi de grau 5 em 6 quadris e de grau 6 em 47 quadris. Em relação à deformidade da cabeça femoral, foram encontradas 10 (19\%) de grau 1, 22 (41\%) de grau 2, e 21 (40\%) de grau 3. Dos 47 pacientes, $35(75 \%)$ apresentavam escoliose na reavaliação final (-Tabela 2).

Achados clínicos: não houve mudança no grau funcional dos pacientes. No total, 25 pacientes (53\%; 29 quadris) apresentaram diminuição ou remissão da dor, mobilidade livre e indolor, e melhora do posicionamento (-Figura 1).

As complicações foram menores em 11 pacientes (23\%; 12 quadris), e 8 pacientes (9 quadris) evoluíram com dor e/ou exposição do material de implante, sendo necessária a sua retirada. 0 tempo médio entre os 2 procedimentos foi de 1 ano e 9 meses, e, após a retirada, os pacientes evoluíram com cicatrização da ferida operatória sem intercorrências ou dor. Dentro deste grupo está um paciente operado bilateralmente, sendo que a exposição do implante ocorreu somente em um dos lados, e o paciente foi considerado neste estudo como sofrendo complicação menor. Dois pacientes apresentaram fratura na diáfise do fêmur ipsilateral, um paciente com redução cruenta e fixação interna com placa e parafusos, e outro com redução incruenta e colocação de gesso; todos evoluíram
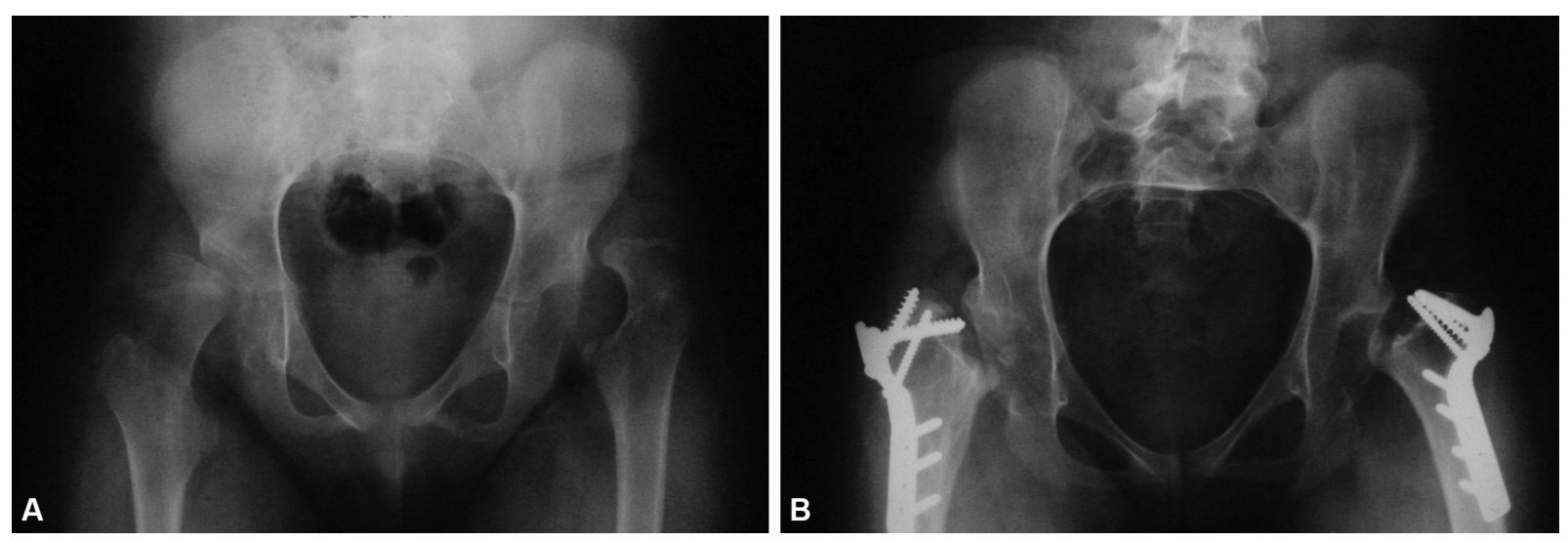

Fig. 1 Paciente do sexo feminino, tetraparética, GMFCS V. (A) Radiografia da bacia no início do acompanhamento com 12 anos e 2 meses de idade. (B) Radiografia da bacia após 4 anos e 2 meses da cirurgia de McHale bilateral aos 16 anos de idade da paciente. 
Tabela 3 Complicações e desfechos

\begin{tabular}{|l|l|l|l|}
\hline & $\begin{array}{l}\text { Sem } \\
\text { complicações }\end{array}$ & Com complicações \\
\hline $\begin{array}{l}\text { Número de } \\
\text { pacientes } \\
\text { (quadris) }\end{array}$ & $25(28)-53 \%$ & $22(24)-47 \%$ & $\begin{array}{l}\text { Complicações maiores } \\
11(12)-23 \%\end{array}$ \\
\hline & & $\begin{array}{l}\text { Complicações menores } \\
11(12)-23 \%\end{array}$ & $6(6)$ retiradas do implante e cirurgias de Castle unilaterais \\
\hline & $8(9)$ retiradas do material de síntese & $3(4)$ retiradas do implante e cirurgias de Castle bilaterais \\
\hline & $2(2)$ fraturas do fêmur ipsilateral & 2 (2) artrodeses do quadril \\
\hline & $\begin{array}{l}1(1) \text { retirada do implante e cirurgia } \\
\text { de Castle contralateral }\end{array}$ & D2-desfecho insatisfatório \\
\hline Desfechos & D1-desfecho satisfatório &
\end{tabular}

satisfatoriamente, com consolidação das fraturas. Um último paciente (1 quadril) evoluiu com luxação dolorosa do quadril contralateral, sendo submetido à cirurgia de Castle; o lado operado com a cirurgia de McHale apresentava-se bem, móvel e sem dor. Portanto, neste desfecho satifatório (D1) da cirurgia de McHale, com ausência de dor, mobilidade dos membros inferiores livre e posicionamento sentado possível na cadeira de rodas, tivemos 36 pacientes (41 quadris), perfazendo 77\% dos nossos casos.

As complicações maiores estiveram presentes em 11 pacientes (12 quadris), dos quais 6 (6 quadris) foram subme- tidos a retirada do implante e cirurgia de Castle unilateralmente, 3 (4 quadris) foram submetidos a retirada do material de implante e posteriormente submetidos a cirurgia de Castle bilateralmente, e 2 (2 quadris) foram submetidos a artrodese do quadril. Destes, 1 caso ( 1 quadril) evoluiu com fratura do fêmur, e foi tratado com fixação externa e posteriormente revisão da artrodese do quadril, e o outro caso foi submetido a duas revisões da artrodese do quadril, e evoluiu sem consolidação, sendo posteriormente realizada a cirurgia de Castle. Dada a falha no procedimento, considerou-se o desfecho insatisfatório (D2) em 11 (23\%) casos (-Tabela 3).
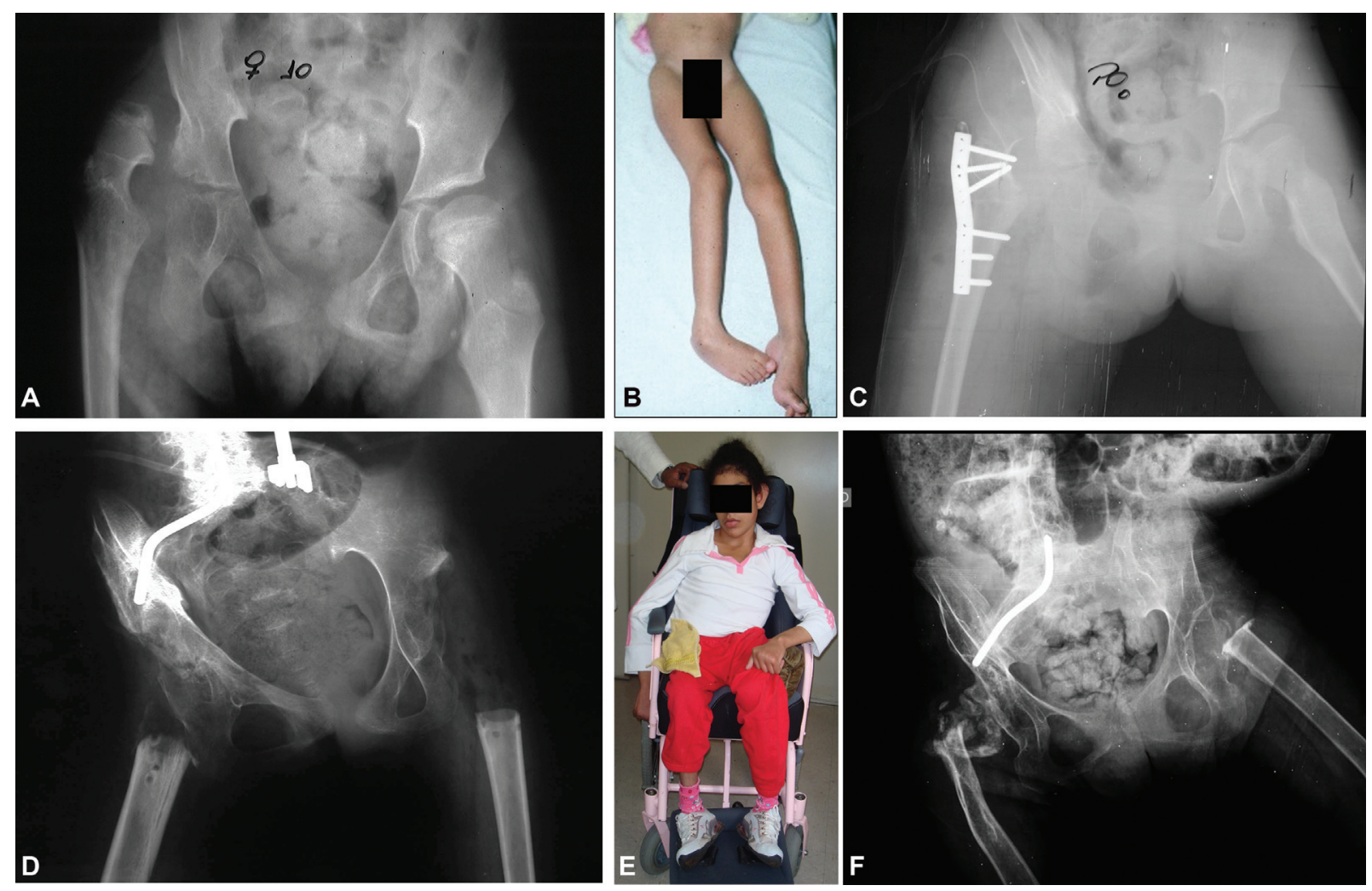

Fig. 2 Paciente do sexo feminino, tetraparética, GMFCS V. (A) Radiografia da bacia no início do acompanhamento com 10 anos e 2 meses de idade. (B) Imagem clínica no início do acompanhamento. (C) Radiografia da bacia no pós-operatório imediato da cirurgia de McHale do lado direito. (D) Radiografia da bacia no pós-operatório imediato da cirurgia de Castle bilateral, aos 14 anos de idade da paciente. (E) Imagem clínica após 7 anos da cirurgia de Castle bilateral, aos 21 anos de idade da paciente. (F) radiografia da bacia após 14 anos da cirurgia de Castle bilateral, aos 29 anos de idade da paciente. 
Não foi evidenciada a presença de ossificação heterotópica nas radiografias pós-operatórias e de migração proximal do fêmur operado.

Os pacientes que persistiram com o quadro álgico foram posteriormente submetidos a cirurgia de Castle, e apresentaram remissão da dor e melhora do posicionamento sentado, mas tal avaliação não é objeto do presente estudo (-Figura 2).

\section{Discussão}

A luxação do quadril é comum, e a incidência está diretamente relacionada à gravidade da espasticidade e ao grau de funcionalidade do paciente. ${ }^{1,3,4,10-13,25}$ A incidência na literatura de dor associada à luxação varia de $25 \%$ a 55\%, sendo de até $90 \%$ nos casos mais graves. ${ }^{1,6,26}$ A mesma incidência foi verificada nos nosso pacientes, uma vez que a maioria deles era de nível funcional IV e V.

Como há presença de escoliose em $79,5 \%$ dos casos, ${ }^{7}$ por vezes torna-se difícil identificar se a dor é decorrente somente da luxação ou da escoliose nestes pacientes graves. A relação entre morfologia do quadril e dor em indivíduos com PC não é clara, e permanece controversa. Alguns estu$\operatorname{dos}^{1,2}$ relataram altos níveis de dor em indivíduos com quadris luxados, com muitos adolescentes necessitando de "cirurgia de salvamento" por conta da perda da articulação do quadril. Os fatores responsáveis pela dor nos quadris deslocados podem incluir a degeneração da cartilagem articular, a resposta inflamatória associada, e a suprarregulação dos mediadores da dor na cápsula da articulação do quadril. ${ }^{27}$ A gravidade e a frequência da dor aumentaram com o aumento da incapacidade física, conforme o GMFCS. ${ }^{8}$ Em relação à $\mathrm{MCPHPS},{ }^{5}$ devido à gravidade dos pacientes e às dificuldades no acesso ao tratamento no nosso sistema de saúde, encontramos 10 quadris de nível 5 e 43 de nível 6, indicando que a cirurgia de salvamento apresentava-se como a única opção de tratamento. A decisão da execução da cirurgia de McHale, salvo nos casos de deformidade grave da cabeça femoral, é também feita no período intraoperatório, quando encontramos mais de $50 \%$ da superfície articular com lesão. Nos casos com lesão entre 30\% e 50\% da superfície articular, nossa conduta é a cirurgia reconstrutora.

Nos casos luxados, geralmente o pequeno trocanter está posicionado à frente da cavidade acetabular, e necessita apenas da valgização femoral proximal. A ressecção da cabeça resulta em melhora da abdução - ao nosso ver, complementa a primeira descrição da osteotomia de valgização intertrocanteriana de Schanz. ${ }^{28}$ A tenodese do ligamento redondo no tendão do psoas, assim como na descrição original, também ao nosso ver dá uma maior estabilidade à construção, e tende a evitar a migração proximal, o que de fato aconteceu nos nossos casos.

Nos nossos pacientes, a deformidade unilateral apresentava uma indicação especial para a cirurgia de McHale, pois, embora seja um procedimento de salvamento, "produz" um aspecto mais harmônico da pelve. Um reflexo disso é a melhora do posicionamento do paciente após a cirurgia, relatado nos nossos casos, assim como nos de outros autores. ${ }^{29}$
Em relação às complicações, na descrição original do procedimento no estudo de McHale et al., ${ }^{15}$ com quatro pacientes, os autores citam a presença de dor no período pós-operatório, acreditando ser ela resultado do atrito da superfície do colo dentro da cápsula articular fechada. Nos nossos casos, os pacientes também mantiveram dor por longo período, em média de seis meses, não sendo encontrada uma explicação anatômica. Faz-se necessário um acompanhamento próximo destes pacientes para o controle medicamentoso, assim como acompanhamento fisioterápico. Nos casos em que a placa utilizada na osteosíntese tornou-se saliente, chegando até a exposição na pele, foi necessária a sua retirada cirúrgica. A exposição da placa geralmente ocorre em pacientes de baixo peso e pouca massa muscular, com pobre suporte nutricional, muitas vezes, no sistema público de saúde, difícil de compensar no período pré-operatório; uma vez tratados com a retirada do material de implante e cuidados locais, os pacientes tornaram-se assintomáticos. Somando-se os pacientes que permaneceram com o implante e os que foram submetidos somente à sua retirada devido à intercorrência da exposição da placa, temos que $77 \%$ de quadris apresentaram boa evolução, sem outras complicações maiores pós-operatórias, a despeito da gravidade funcional do grupo de pacientes tratados.

Outra complicação não rara nos pacientes GMFCS V é a fratura no membro inferior: pacientes não deambuladores, com visível baixa massa óssea (embora não medida) e a presença de contraturas articulares, somadas a eventuais movimentos bruscos durante o cuidado com o paciente, representam fatores de risco importantes para fraturas. A depender do tipo e da localização da fratura, o tratamento pode ser incruento ou cruento, considerando-se sempre o aspecto funcional do paciente. $O$ episódio da fratura não implica que, após o tratamento desta, o objetivo da cirurgia de McHale não tenha sido alcançado. Por esse motivo, as fraturas foram consideradas complicações menores.

Para alguns pacientes, a dor representou um fator limitante importante para os cuidados diários, mesmo após o procedimento de McHale. Para estes casos, houve a necessidade de ampliar a ressecção e convertê-la em cirurgia de Castle. O objetivo era sempre de alívio da dor e melhora do posicionamento do paciente.

Outra opção nas duas pacientes após a falha na osteotomia de McHale foi a artrodese do quadril, sendo que, na primeira paciente, de nível funcional $\mathrm{V}$, tentou-se a estabilização do quadril e melhora da dor. Porém, na evolução, a paciente apresentou piora da deformidade vertebral e, após a artrodese vertebral, tornou-se incompatível o posicionamento sentada, e a paciente foi posteriormente submetida à cirurgia de Castle bilateralmente, com boa evolução. A outra paciente, de nível funcional IV, a despeito de apresentar fratura do fêmur ipsilateral, tratada com fixador externo, apresentou e ainda mantém um bom posicionamento.

Concordamos com a literatura ${ }^{6,19,20,30}$ que o tratamento cirúrgico para o quadril espástico doloroso e luxado no contexto de PC não é perfeito. Permanece uma grande porcentagem de falhas, apesar das numerosas técnicas cirúrgicas desenhadas para tratar este problema. Devemos 
considerar que, na literatura, mesmo com a utilização do GMFCS como padrão de avaliação, a homogeneidade quanto à espasticidade dos pacientes no mesmo nível funcional fica pouco clara. Ademais, são pacientes que geralmente apresentam comorbidades que influenciam o desfecho de qualquer tratamento cirúrgico.

Este estudo apresenta algumas limitações. Por ter sido baseado em análise de prontuários, não foi possível reavaliar os pacientes quanto à dor utilizando escalas validadas. Também não foi aplicado questionário para análise da qualidade de vida dos pacientes. Relatos nos prontuários médicos incluíam a descrição do exame físico, presença ou não da dor à movimentação do quadril, e as dificuldades do posicionamento do paciente. Por outro lado, todos os pacientes foram e são tratados pela mesma equipe médica, com o mesmo protocolo de tratamento, o que de alguma maneira uniformiza a avaliação. Estudos prospectivos priorizando a análise do impacto na qualidade de vida são necessários.

\section{Conclusão}

Mediante os resultados deste trabalho, demonstramos que a cirurgia de McHale é uma opção no tratamento do quadril doloroso da PC espástica com nível funcional IV e V, com melhora quanto à dor e ao posicionamento do paciente, mas devemos estar preparados para a abordagem das possíveis complicações, como as fraturas e a persistência da dor.

\section{Conflito de Interesses}

Os autores declaram não haver conflito de interesses.

\section{Referências}

1 Miller F. Cerebral Palsy. New York: Springer Science; 2005

2 Horstmann HM, Bleck EE. Orthopaedic Management in Cerebral Palsy. London: MacKeith Press; 2007:344-379

3 Robin J, Graham HK, Baker R, et al. A classification system for hip disease in cerebral palsy. Dev Med Child Neurol 2009;51(03):183-192

4 Robin J, Graham HK, Selber P, Dobson F, Smith K, Baker R. Proximal femoral geometry in cerebral palsy: a population-based crosssectional study. J Bone Joint Surg Br 2008;90(10):1372-1379

5 Howard JJ, Khot A, Graham HK. The hip in cerebral palsy. In: Alshryda S, Howard J, Huntley J, Schoenecker J, eds. The pediatric and adolescent hip. Basel, Switzerland: Spinger Nature Switzerland; 2019:467-530

6 Wright PB, Ruder J, Birnbaum MA, Phillips JH, Herrera-Soto JA, Knapp DR. Outcomes after salvage procedures for the painful dislocated hip in cerebral palsy. J Pediatr Orthop 2013;33(05):505-510

7 Knapp DR Jr, Cortes H. Untreated hip dislocation in cerebral palsy. J Pediatr Orthop 2002;22(05):668-671

8 Wawrzuta J, Willoughby KL, Molesworth C, et al. Hip health at skeletal maturity: a population-based study of young adults with cerebral palsy. Dev Med Child Neurol 2016;58(12):1273-1280

9 Terjesen T. The natural history of hip development in cerebral palsy. Dev Med Child Neurol 2012;54(10):951-957

10 Shore BJ, Yu X, Desai S, Selber P, Wolfe R, Graham HK. Adductor surgery to prevent hip displacement in children with cerebral palsy: the predictive role of the Gross Motor Function Classification System. J Bone Joint Surg Am 2012;94(04):326-334

11 Shore BJ, Graham HK. Management of moderate to severe hip displacement in nonambulatory children with cerebral palsy. JBJS Rev 2017;5(12):e4

12 Ramstad K, Terjesen T. Hip pain is more frequent in severe hip displacement: a population-based study of 77 children with cerebral palsy. J Pediatr Orthop B 2016;25(03):217-221

13 Silverio AL, Nguyen SV, Schlechter JA, Rosenfeld SR. Proximal femur prosthetic interposition arthroplasty for painful dislocated hips in children with cerebral palsy. J Child Orthop 2016;10(06): 657-664

14 Castle ME, Schneider C. Proximal femoral resection-interposition arthroplasty. J Bone Joint Surg Am 1978;60(08):1051-1054

15 McHale KA, Bagg M, Nason SS. Treatment of the chronically dislocated hip in adolescents with cerebral palsy with femoral head resection and subtrochanteric valgus osteotomy. J Pediatr Orthop 1990;10(04):504-509

16 Girdlestone GR. Acute pyogenic arthritis of the hip: an operation giving free access and effective drainage. Lancet 1943; 241:419-421

17 Fucs PM, Yamada HH. Hip fusion as hip salvage procedure in cerebral palsy. J Pediatr Orthop 2014;34(Suppl 1):S32-S35

18 Root L, Goss JR, Mendes J. The treatment of the painful hip in cerebral palsy by total hip replacement or hip arthrodesis. J Bone Joint Surg Am 1986;68(04):590-598

19 Kolman SE, Ruzbarsky JJ, Spiegel DA, Baldwin KD. Salvage options in the cerebral palsy hip: a systematic review. J Pediatr Orthop 2016;36(06):645-650

20 de Souza RC, Mansano MV, Bovo M, et al. Hip salvage surgery in cerebral palsy cases: a systematic review. Rev Bras Ortop 2015;50 (03):254-259

21 Givon U. Management of the spastic hip in cerebral palsy. Curr Opin Pediatr 2017;29(01):65-69

22 Reimers J. The stability of the hip in children. A radiological study of the results of muscle surgery in cerebral palsy. Acta Orthop Scand Suppl 1980;184(184):1-100

23 Murphy RF, Mooney JF III. Current concepts in neuromuscular scoliosis. Curr Rev Musculoskelet Med 2019;12(02):220-227

24 Palisano R, Rosenbaum P, Walter S, Russell D, Wood E, Galuppi B. Development and reliability of a system to classify gross motor function in children with cerebral palsy. Dev Med Child Neurol 1997;39(04):214-223

25 Flynn JM, Miller F. Management of hip disorders in patients with cerebral palsy. J Am Acad Orthop Surg 2002;10(03): 198-209

26 Soo B, Howard JJ, Boyd RN, et al. Hip displacement in cerebral palsy. J Bone Joint Surg Am 2006;88(01):121-129

27 Masłoń A, Jóźwiak M, Pawlak M, Modrzewski T, Grzegorzewski A. Hip joint pain in spastic dislocation: aetiological aspects. Dev Med Child Neurol 2011;53(11):1019-1023

28 Schanz A. Zur Behandlung der veralteten angeborenen Hüftverrankung. Munch Med Wochenschr 1922;23:930-931

29 Leet AI, Chhor K, Launay F, Kier-York J, Sponseller PD. Femoral head resection for painful hip subluxation in cerebral palsy: Is valgus osteotomy in conjunction with femoral head resection preferable to proximal femoral head resection and traction? J Pediatr Orthop 2005;25(01):70-73

30 Boldingh EJ, Bouwhuis CB, van der Heijden-Maessen HC, Bos CF, Lankhorst GJ. Palliative hip surgery in severe cerebral palsy: a systematic review. J Pediatr Orthop B 2014;23(01): 86-92 